\title{
Efficiency of Artificial Neural Networks (Percepton Network) in the Diagnosis of Thyroid Diseases
}

\author{
Suher A.Dawood \\ College of Administration \\ and Economics \\ University of Mosul, Iraq \\ Laheeb M. Ibrahim \\ College of Computer \\ Sciences Mathematics
}

\section{Received on: 18/08/2002}

\section{ABSTRACT}

Thyroid gland software which was obtained through research is considered an effective system to diagnosed thyroid gland automatically. This is done by a built complementary database which is flexible and easy at work with data patients concerning those patients under observation at Hazim Al-Hafith Hospital for Oncology \& Nuclear Medicine in Mosul. The activity of Thyroid gland software was tested on information about 200 Patients, and information about them was stred in Thyroid database, after that we diagnosed The Thyroid Gland Disease by using an artificial neural network (Perceptron) that is able to recognize Thyroid Gland Disease in good recognized ratio and with a ratio close to the doctor diagnosis depending on (sign \& symptoms) which may enables the doctors in depending on it the right diagnosis for the disease.

Keyword: artificial neural network, Perceptron , Thyroid

$$
\begin{aligned}
& \text { كفاءة الشبكات العصبية الاصطناعية (شبكة المدرك) في تثخيص أمراض الغدة الدرقية }
\end{aligned}
$$

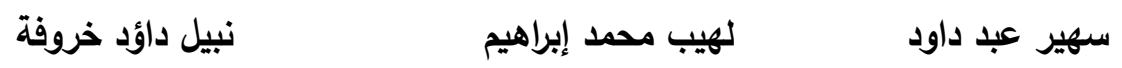

$$
\begin{aligned}
& \text { كلية الإدارة والاقتصاد كلية علوم الحاسبات والرياضيات مستثفى حازم الحافظ للأورام } \\
& \text { والطب النووي } \\
& \text { تاريخ قبول البحث: 2003/01/04 } \\
& \text { جامعة الموصل لافل } \\
& \text { تاريخ استلام البحث: 2002/08/18 } \\
& \text { الملخص } \\
& \text { يُعد نظام الغدة الدرقية(Thyroid) المنجز خلال البحث وسيلة ذات فاعلية في متابعة } \\
& \text { مسيرة مرضسى الغدة الدرقية متابعة آلية وذلك من خـلال بناء قاعدة بيانات متكاملة توفر المرونة } \\
& \text { والسهولة للتعامل مع معلومات المرضى الحقيقية التي تم أخذها من مستثفى حازم الحافظ للأورام } \\
& \text { والطب النووي في الموصل. كما يتضمن البحث بناء وحدة التثخيص الطبي التي استخدمت أسلوب التهاب } \\
& \text { الثبكة العصبية الاصطناعية المدرك(Perceptron) في تثخيص أمراض الغدة الدرقية و أظهرت }
\end{aligned}
$$

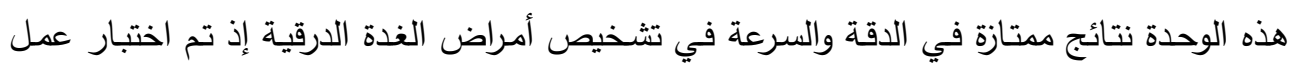

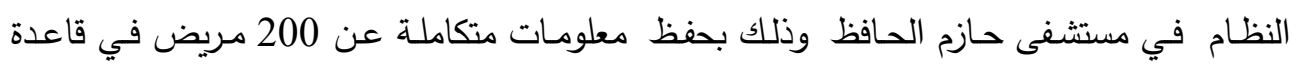


البيانات المبنية في النظام ومن ثم تثخيص الحالات المرضية لمرض الغدة الدرقية باستخدام الثبكة العصبية الاصطناعية، إذ استطاعت الثبكة تمييز الحالات المرضية بنسبة نجاح جيدة وبذلك يمكن القول انه ممكن تدريب الشبكات العصبية لإعطاء تشخيص صحيح لإمراض الغدة الدرقية وبنسبة مقاربة لتثخيص الطبيب بالاعتماد على العلامات والأعراض السريرية التي تمكن

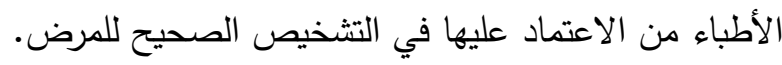
الكلمات المفتاحيه: الشبكات العصبيه الاصطناعيه، شبكه الددرك، الغدة الدرقية

1 1 ـ المقدمة

نتيجة للتطورات الهائلة التي يشهدها العالم في الميادين والنشاطات المختلفة وخاصـة في

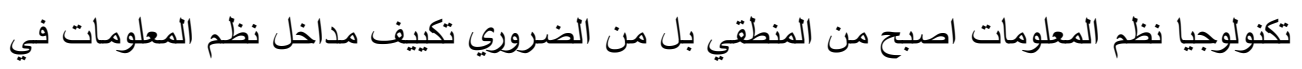

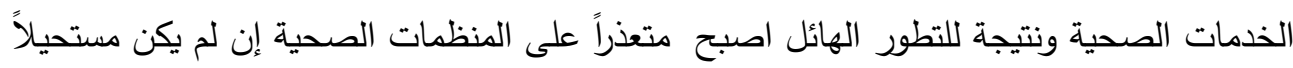
عليها معارضة التخطيط لاستخدام مواردها وجدولة عمليات إنجاز الخدمة بواسطة الحاسوب. ونظئهرا

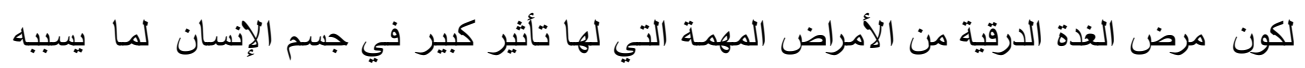

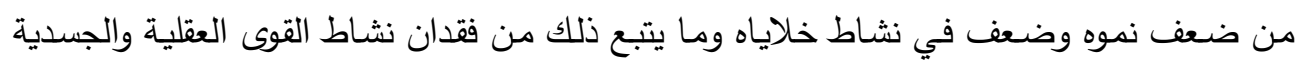

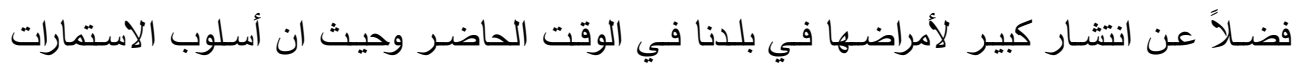

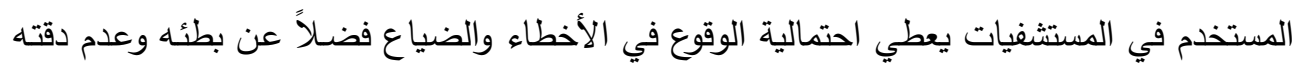

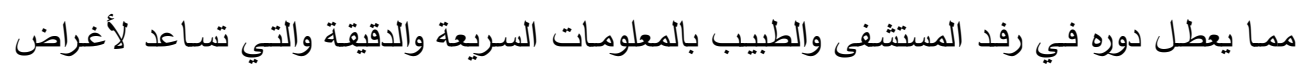
التثخيص والعلاج والبحث العلمي اصبح استخدام الحاسوب أمرا ضروريا ـ ونظرا لقدرة علم تمييز

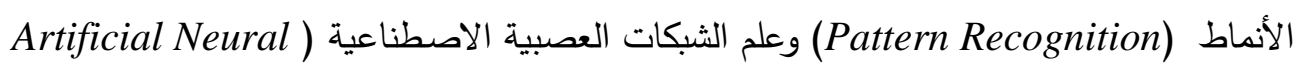

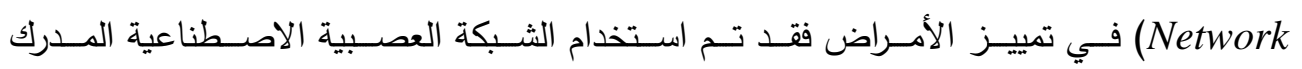
الإZurad,1996] لتشخيص مرض الغدة الدرقيـة إذ يعد التصنيف إحد أهم

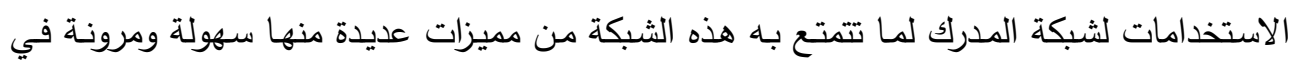

التعامل وملاءمتها للبيانات [ اسطيفان ، Werner, 1995,1991].

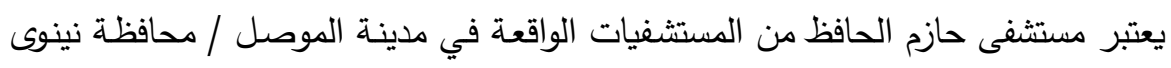

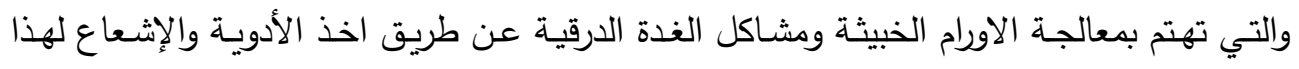

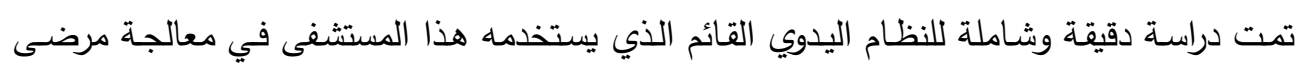

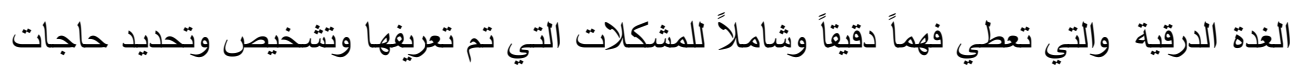

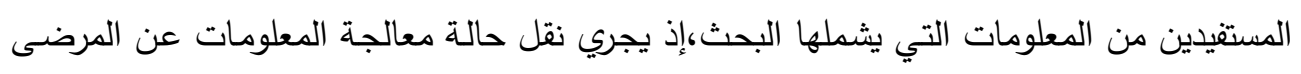

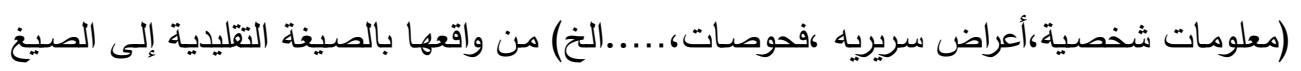


الحاسوبية فضـلاً عن بناء وحدة تشخيص طبي لمرض الغدة الدرقية باستخدام الثبكة العصبية

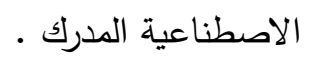

أن الهدف الذي ننشده من البحث هو تصميم نظام آلي لنقل حالة معالجة المعلومات عن

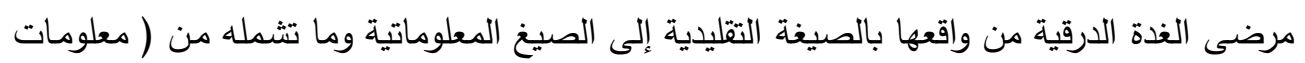

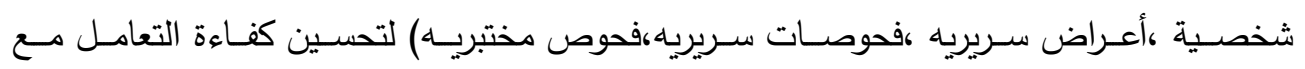
المعلومات مع مراعاة (السرعة ،الدقة،حفظ المعلومات بصورة آمنة ) والاستفادة من ميزات علم تمييز لتحئ

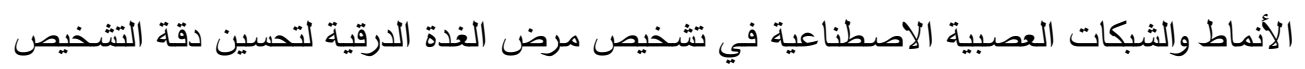

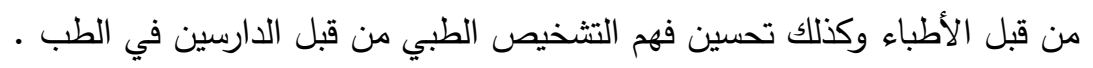

\section{Thyroid Gland}

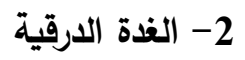

الغدة الدرقية هي إحدى الغدد الصماء الموجودة في جسم الإنسان ، وهي اكبر

الغدد الصماء حجماً وتقع في مقدمة العنق تحت الحنجرة وتغطي مقدمة القصبة الهوائية وتتكون

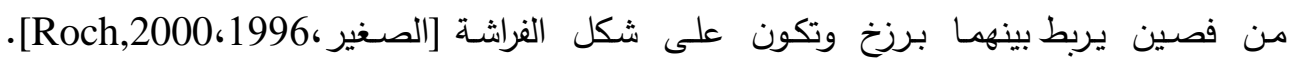

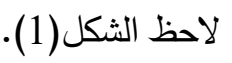

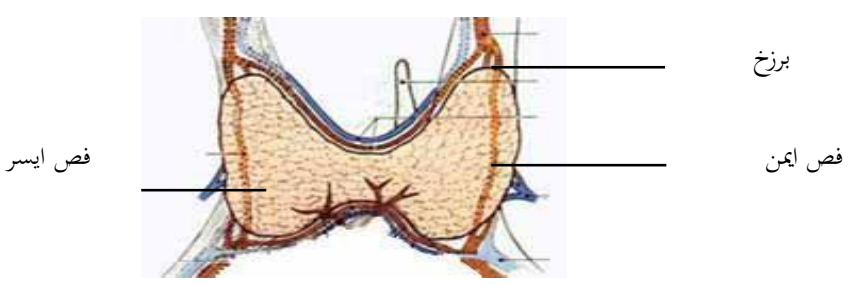

الشكل (1) الثكل العام للغدة الدرقية.

يمكن تمييز تضخم الغدد الدرقية بسهولة وذلك بحكم موقعها في مقدمة العنق مما يؤدي إلى فقدان الثكل المتتاسق للعنق وبعض الناس يعتبر هذا التضخم تثويهًا أو عاهة جسدية وسبب هلته

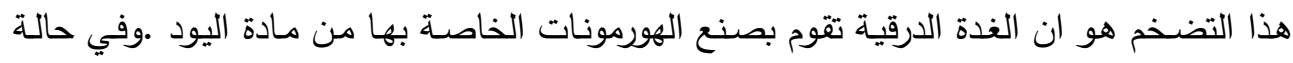

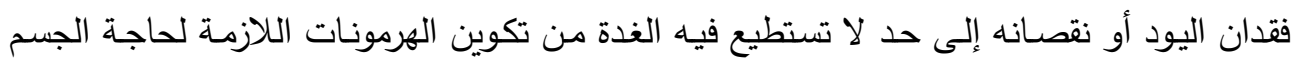

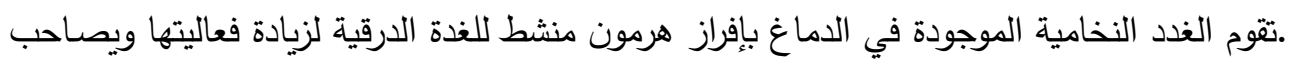

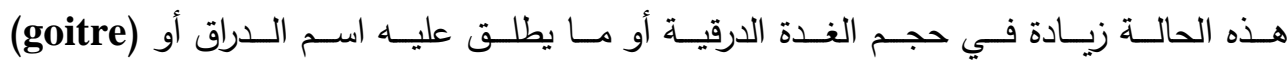

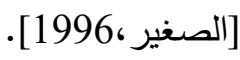




\section{3- نظرة تاريخية في التثخيص الطبي والثبكات العصبية:}

يُعد التثخيص الطبي (تمييز الأمراض) أحد أهم تطبيقات الثبكات العصبية الاصطناعية، حيث تعتبر التصانيف (Classification) من أهم هذه التطبيقات، ولان تحديد نوع المرض يعتمد على التصنيف وهو جانب مهم من جوانب استخدامات الثبكة العصبية، فهنا تكمن طبيعة العلاقة بين الثبكات العصبية والجانب الطبي، وعادة يتم استخدام الشبكات العصبية المدربة بإشراف في الجانب الطبي لان عملية التدريب تحتاج إلى أزواج التدريب (الإدخـال والهـدف) او (الإدخـال والإخراج) وكلاهما يتم تحديدهما مسبقاً لغرض تخمين المتغيرات الداخلية (الأوزان)[Eric,1995]. ومن خلال الاطلاع على الدراسات والبحوث المنجزة في مجال استخدام الثبكات العصبية الاصطناعية في التشخيص الطبي وجد أن الثبكات العصبية لها دور كبير في التشخيص الصحيح للمرض بشكل كفوء ومقارب لتشخيص الطبيب المتخصص ـ فقد تم استخدام الثبكة العصبية (Hamming) يتراوح عدد كريات الدم البيضاء في دم الإنسان بين 1000-15000 كرية دم بيضاء في المليمتر

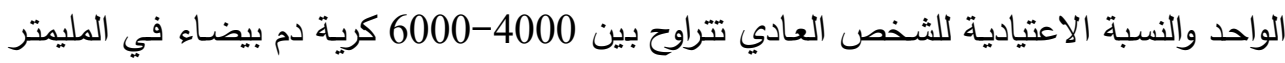
الواحد وأي خلل من ارتفاع و انخفاض سيؤدي إلى إصابة الإنسان بعدد من الأمراض وكانت نتأنتائج

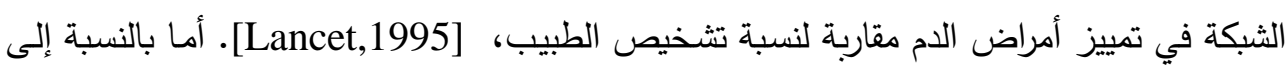
تثخيص أمراض الغدة الدرقية باستخدام الثبكات العصبية الاصطناعية ففي الحقيقة يعتبر البحث

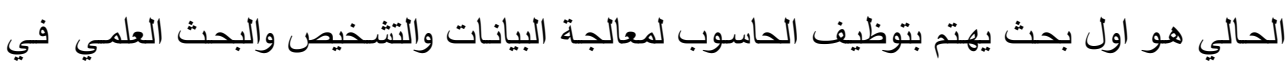
مستثفى حـازم الحافظ وقد تم استخدام شبكة المدرك (Perceptron) في تمييز أمراض الغدة

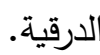

\section{4- الثبكة العصبية الاصطناعية المدرك (Perceptron)}

يمكن تعريف الثبكات العصبية الاصطناعية (Artificial Neural Network ANN) على أنها العلم الذي يهتم بدراسة أنظمة الضبط والاتصالات في الكائنات الحية، بغية صنيه الاصنع نموذج

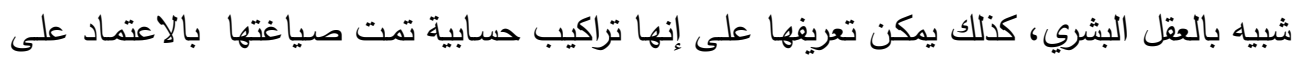

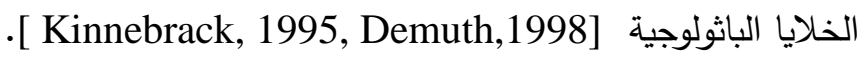


شبكة المدرك تعتبر واحدة من الثبكات العصبية الاصطناعية الثائعة الاستعمال ويعتبر

العالم روزنبلات مكتشف هذه الثبكة في عام (1958) ،تتكون الشبكة من طبقتين طبنة طبنة الإدخال

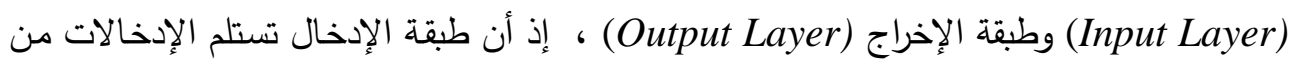

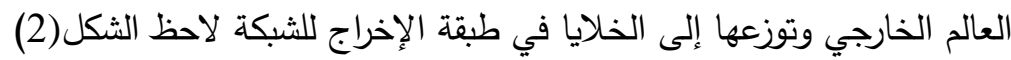

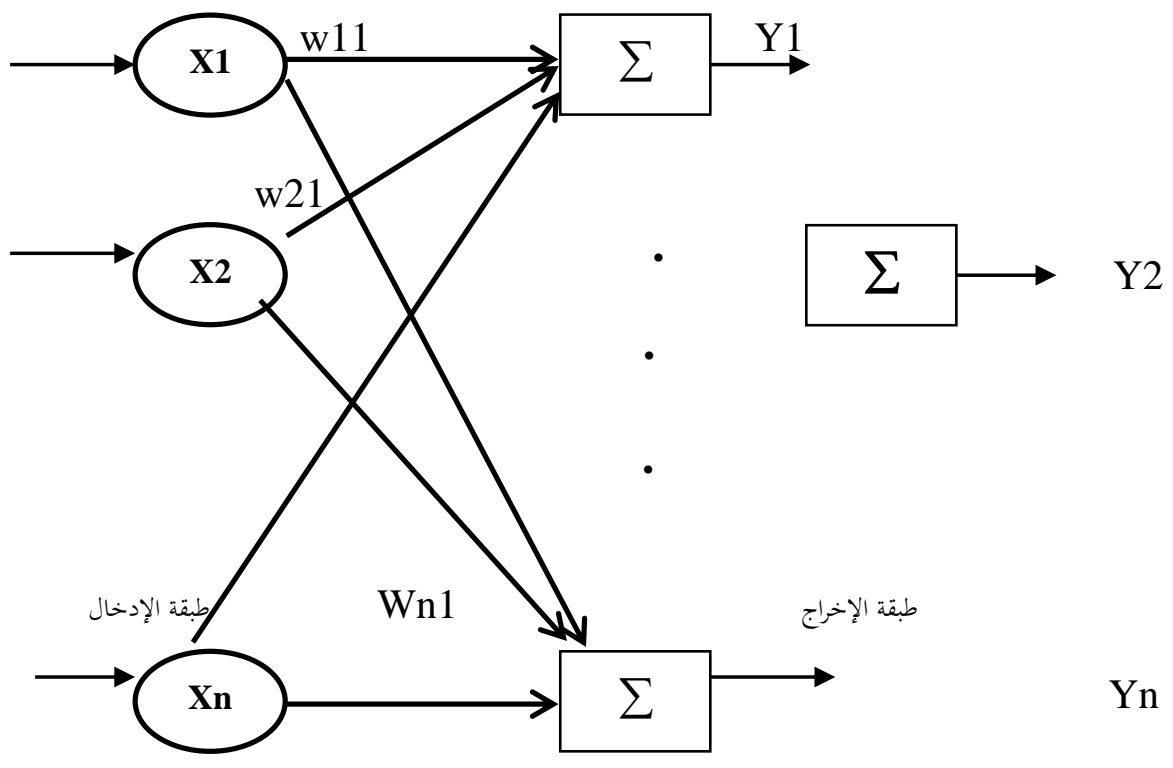

الثكل (2) شبكة المدرك (Perceptron) ذات الطبقة الواحدة

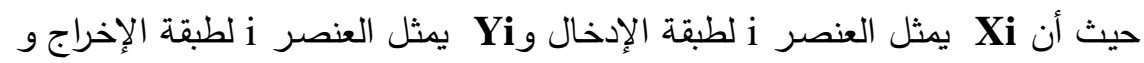

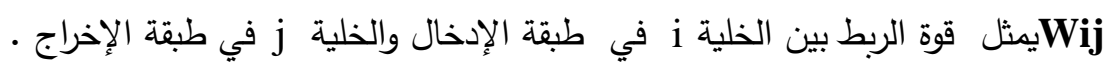

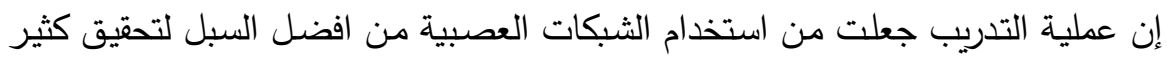

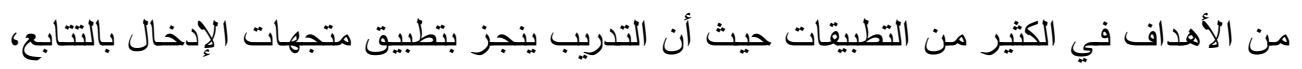

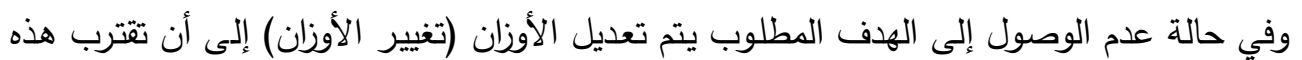

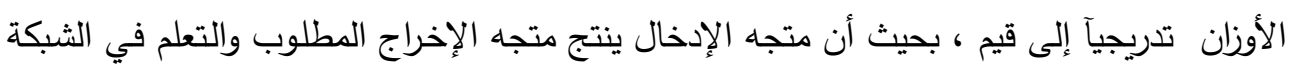

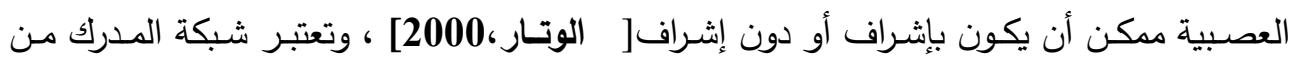

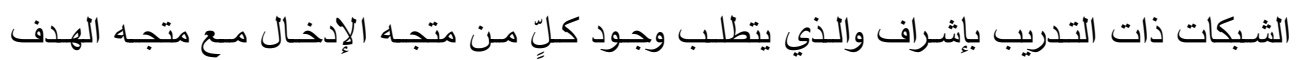

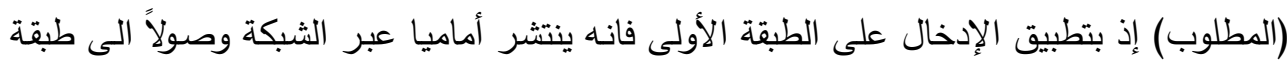

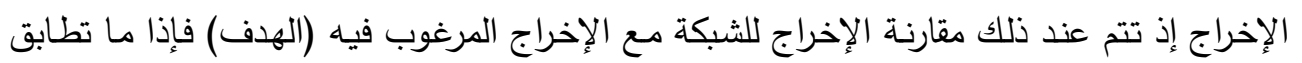


الاثنان يعني أننا وصلنا إلى الحل الصحيح وإذا لا نعيد تدريب الثبكة بتغيير الأوزان إذ أن الأوزان تغير طبقاً إلى خوارزمية تهدف إلى تقليل الخطأ [ياسين،2000].

\section{Thyroid Tلتيل وتصميم قاعدة بيانات نظام}

إن تحليل النظم يعني تحويل نظام يدوي لمؤسسة قائمة إلى نظام حاسوبي،لذا ستكون عملية

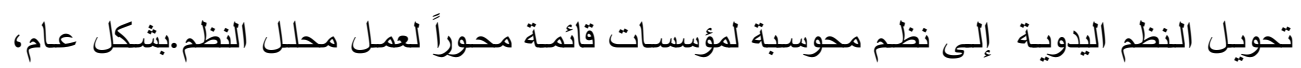

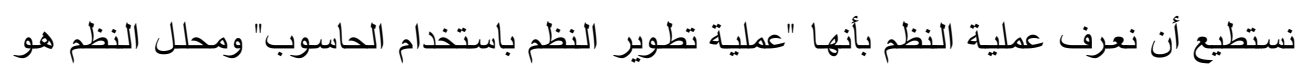

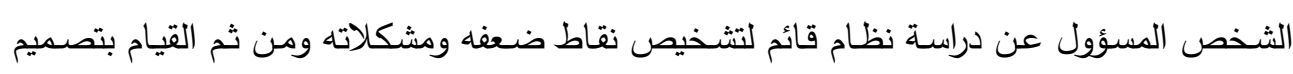
نظسام جديد وتجريبه،ويمكن تصنيف الوظـائف المناطـة بمحلـل النظم بالدراسـة التمهيديـة للنظـام الحالي (اليدوي)، مرحلة الدراسة التفصيلية(تحديد متطلبات النظام)،مواصفات اللغة المستخدمة لبناء

النظام، التصميم التفصيلي للنظام، أمنية النظام، [الزبيدي, 1998 ، علي، 1989]

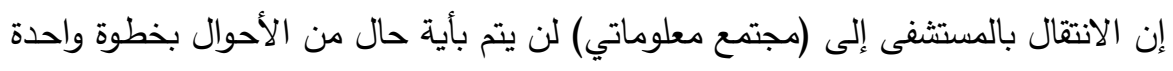
، بل أن الأمر يحتاج إلى اكثر من ذلك بكثير فليست الغاية من الوصول إلى (مجتمع معلوماتي) باتئي تكديس الوسائل والتتنيات بل الانتقال التدريجي الهادي والمدروس ، وبتوفير (البيئة) المعلوماتية لئية

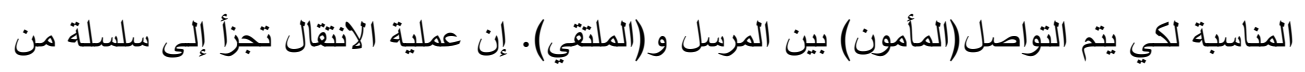

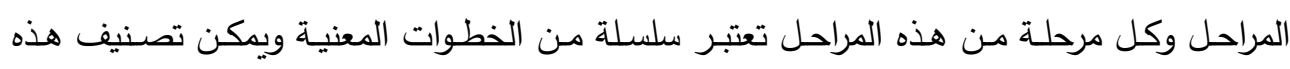

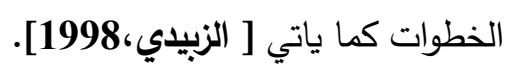

مرحلـة الاراسـة التمهيديـة: تعتبر المرحلة التمهيدية المرحلة الأولى في تحليل وتصميم نظام thyroid

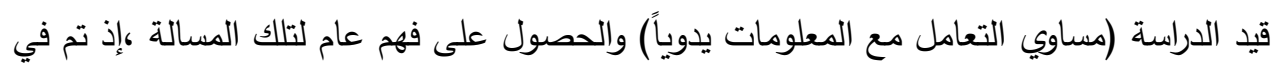

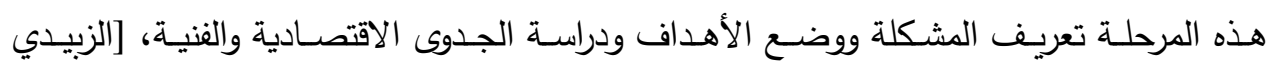
1998، وقد تم تحديد المشكلة بما ياتي:(إن استخدام الأسلوب التقليدي في التعامل مع معلومات

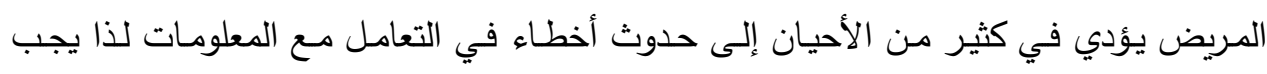

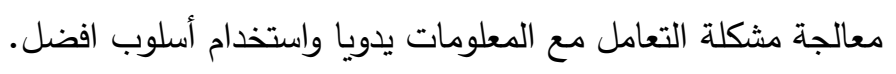
مرحلة الاراسة التفصيلية(تحديد المتطلبات) : تم في هذه المرحلة دراسة دقيقة وشاملة لنظام الغدة

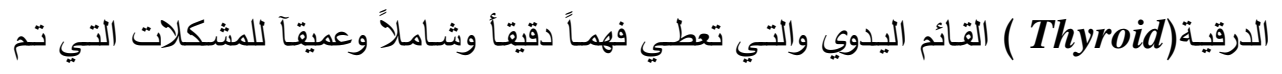

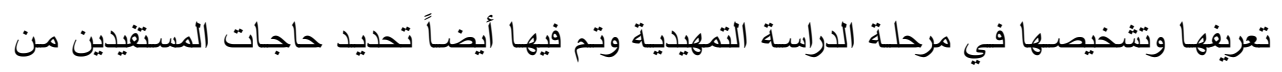

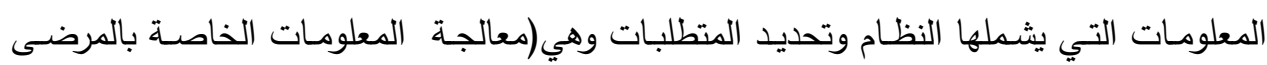


(إضـافة ،تحديث،عرض،تثـخيص،تقارير ...الـخ) ، سـهولة البحث والحصـول على إحصـائيات

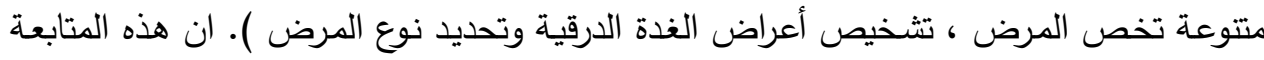

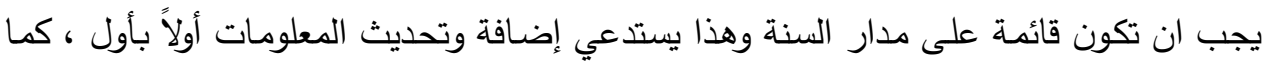

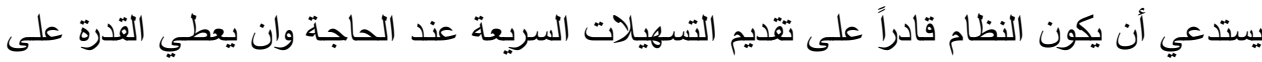
تقصي حالة المرض في أي وقت. لتكون.

التصـيم التفصيلي للنظام: هناك عدد من النقاط تم اخذها بنظر الاعتبار عند البدء بتصميم نظام (Thyroid ) هي ان يكون امتداداً للنظام التقليدي (اليدوي) المتبع في مستشفى حسازم الحافظ للاورام والطب النووي بحيث تم استخدام استمارات المعلومات المتوفرة في مستثفى حازم الحافظ مع بعض التعديلات و سهولة استخدامه من قبل العاملين وذلك بتصميم واجهات صورية وسهولة احتواء التغييرات واستخدام الملفات وان تكون الاحتياطات الأمنية وسرية المعلومات عالية

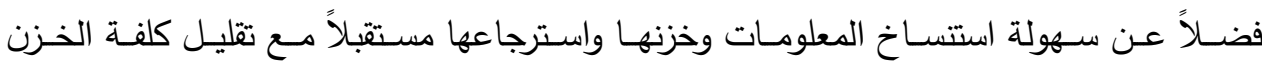
والاسترجاع وزيادة الدقة في إدخال المعلومات وتقليل التكرارية. تم في هذه المرحلة تصميم مفصل

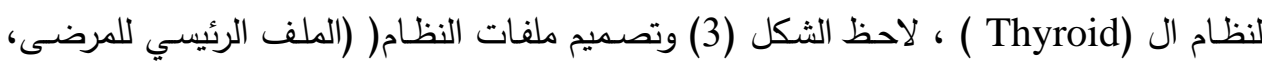

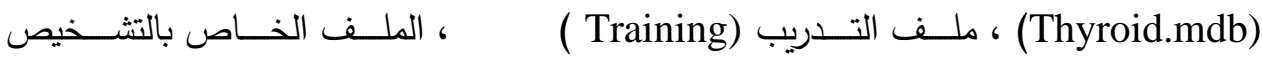

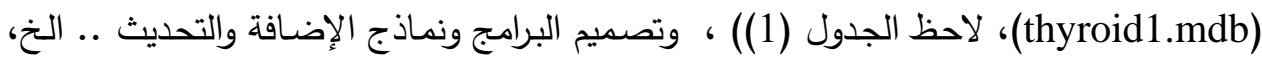
لاحظ الثكل (4) ونمـاذج الإخراج والتقاريير (استخدام التقارير في هذا النظام يعد من الفعاليات المهمة في النظام وذلك لتحديد كفاءة الأداء ولإجراء الإحصائيات الضرورية في تقييبي فعالية

النظام ولإجراء البحوث.

\begin{tabular}{|c|c|c|c|}
\hline المواصفات & الحجم & النوع & اسم الحقل \\
\hline اسم المريض & 80 & Text & Patient \\
\hline نوع المرض الذي تم تحديده من & 15 & Text & Diagonsis \\
\hline
\end{tabular}

جدول (1) ملف التثخيص للمريض (thyroid1.mdb)

6

ابتداءً من المؤتمر الأول للتشخيص الطبي المسند بالحاسبة الإلكترونية المنعقد في مدينة Dyou 
(IMIA) ذلك الحين اصبح التشخيص الطبي المسند بالحاسبة الإلكترونية يجتذب اهتمام الباحثين خصوصاً بعد الزخم الذي اكتسبه هذا الاتجاه في العقد الأخير عند ظهور نجم الثبكات العصبية الاصطناعية

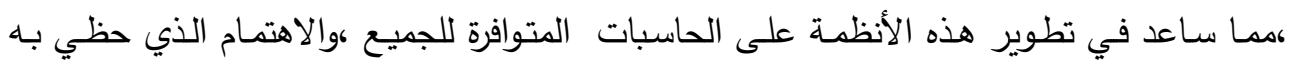

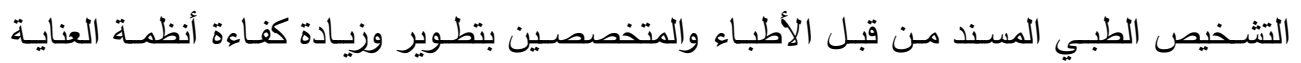
الطبية[Dats, 1998] من المعروف أن لكل مرض مجموعـة من الأعراض المعروفة طبياُ بصورة جيدة وتعني كلمـة

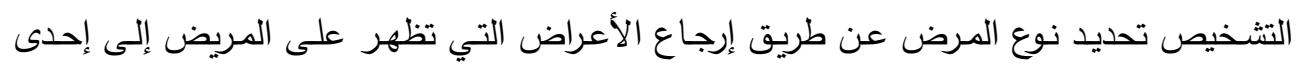

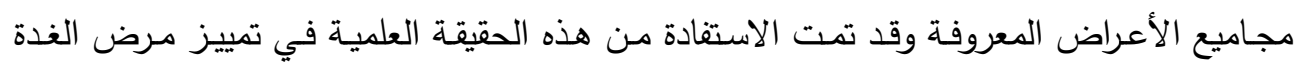

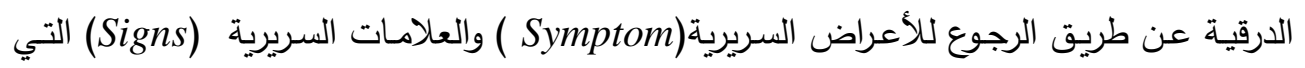

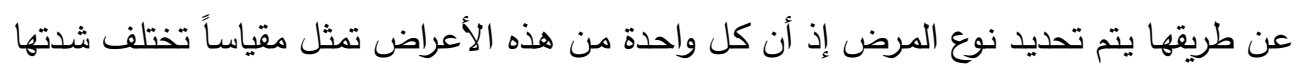
حسب تأثيرها في المرض.

مدخلات شبكة المدرك: تعتبر الأعراض والعلامات السريرية (Symptom \& signs) أهم مدخلات شبكة المدرك(Perceptron) • إذ يتم طرح مجموعة من الأسئلة على المريض تمثل

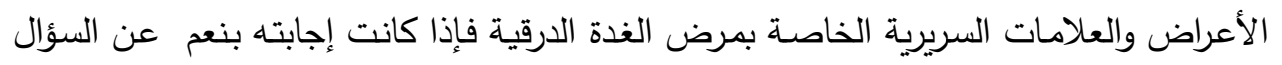

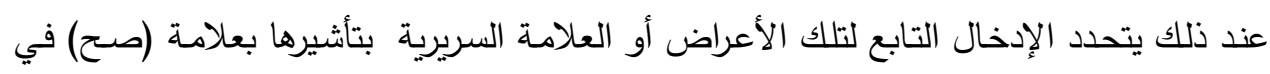
صندوق الاختبار (Check box)، لاحظ الثكل( 4) داخل الثكل الخاص بملف (Thyroid) حيث تمثل ب "1" داخل الملف وإذا كانت إجابة المريض ب(لا) لاحف عن السؤال يتم خزن "صغر" في الحقل المخصص داخل ملف Thyroid وهكذا بالنسبة إلى باقي الأسئلة والأعراض السريرية

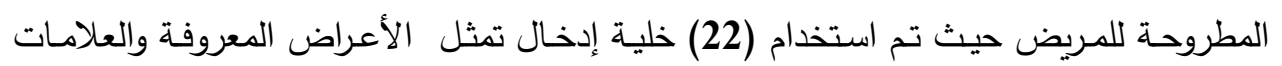
السريرية (Symptoms \& signs) التي تخص مرض الغدة الدرقية وبقيم تتراوح بين صفر وواحد

مخرجات شبكة المدرك : تم اختيار (3) خلايا في طبقة الإخراج لشبكة المدرك (Perceptron)

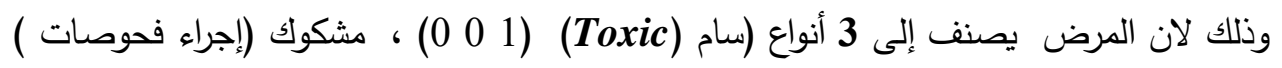
(Equivocal) الإخراج تستلم مدخلاتها من كل خلية في طبقة الإدخال وحسب أهمية هذه المدخلات وشدة تأثيرها في ظهور المرض سوف يتم تصنيف المرض. 
تدريب شبكة المدرك على تصنيف مرض الغدة الارقيـة : إن استجابة الثبكة للتصنيف يتم تحديدها عن طريق تدريب الثبكة العصبية على مجموعة من الأوزان يتم تدريب الثبكة عليها لانتاج الإخراج المطلوب الذي يتم تمريره على دالة العتبة (Threshold-Function) التي تعمل

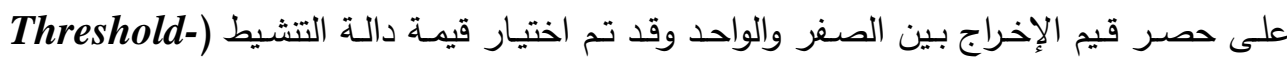
(value ذلك حساب مقدار الخطأ حيث يقارن مع شرط الاحتمال (Tolerance) فإذا كان مقدار الإحراج الخطأ اكبر من شرط الاحتمال الذي تم أخذه (0.1) سوف يستمر التدريب بتغيير الأوزان وتدريب الثبكة

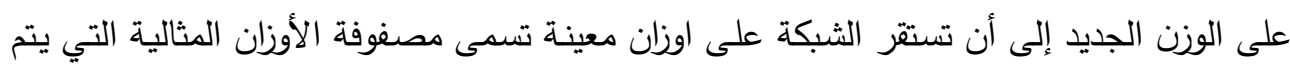

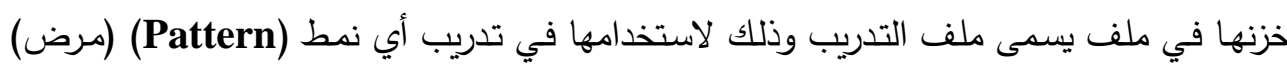
يدخل إلى الشبكة. الاختبار : ان اختبار عمل الثبكة يعتبر ضرورياً للتعرف على قدرة الثبكة على تمييز المرض فإذا

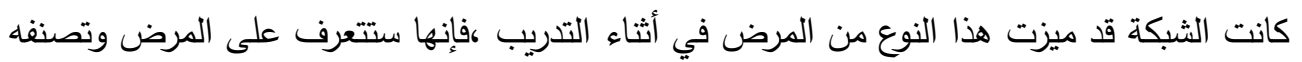
إلى نوع معين ويتم خزن نوع التصنيف في حقل (diagnosis) والمريض الذي لم تتعرف الثبكة على نوع مرضه سوف يخزن في حقل ( diagnosis) عبارة (غير موجود) وتتم عملية الاختبار في

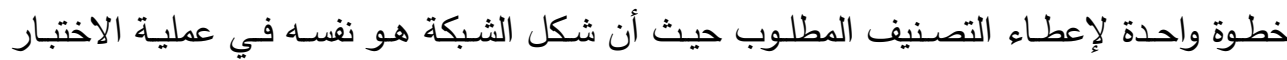
والتدريب وممكن توضيح النتائج التي تم الحصول عليها من عملية التدريب في الجدول (2).

\begin{tabular}{|c|c|c|c|}
\hline عدد المحاولات & نسبة الخطأ & نسبة التعلم & الصنف \\
\hline 10 & $\mathbf{0}$ & 0.0002 & سام (Toxic) \\
\hline 50 & $\mathbf{0}$ & 0.0002 & إجراء فحوصات(Euqivocal) \\
\hline 10 & $\mathbf{0}$ & 0.0002 & غير سام (non toxic) \\
\hline
\end{tabular}

الجدول (2) يمثل النتائج النهائية لتدربب شبكة المدرك (Percepton) في تمييز مرض الغدة

\section{الارقية}

7- الاستنتاجات

إن الغاية الأساسية من تصميم نظام الغدة الدرقية هو توفير وسيلة ذات فاعلية للمساعدة في متابعة مسيرة المرضى متابعة آلية والتقدم خطوة نحو تحويل هذه المتابعة من الصيغة التقليدية

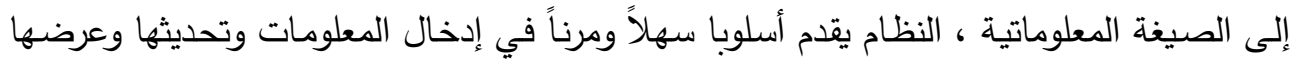

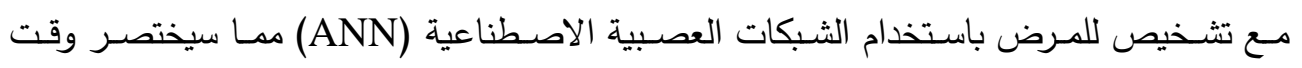
الإنجاز من دون شك ويعطي إنتاجية عالية من خلال ما ياتي:- 
تم تدريب الثبكة باستخدام (17) حالة مرضية لمختلف الأمراض وقد استطاعت الثبكة التدريب على جميع الحالات المرضية وتصنيفها بالثكل الصحيح.

تم اختبار عمل الشبكة العصبية الاصطناعية المستخدمة في تشخيص مرض الغدة الدرقية وبذلك باختيار (200) مريض تم عرضهم على الثبكة إذ استطاعت الشبكة تمييز الحالات

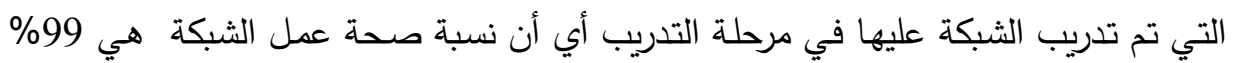

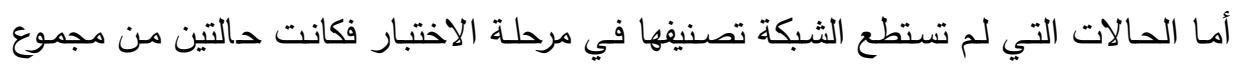
(200) حالة وذلك لان الشبكة لم تتدرب عليها في مرحلة التدريب وبذلك يمكن القول انه ممكن

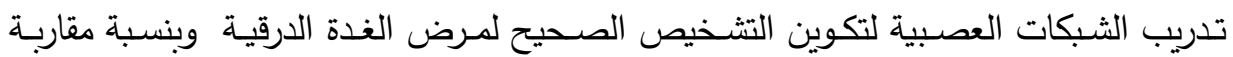

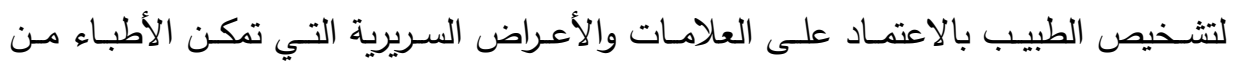
الاعتماد عليها في التثخيص الصحيح للمرض.

مميزات النظام نظام الغدة الدرقية يوفر وسيلة للتعامل مع المعلومات الأساسية الخاصة بالمرض من خلال

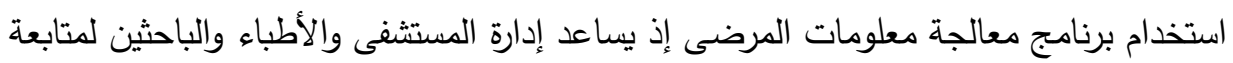
المرضى وإصدار الإحصائيات.

توفير مؤشرات رقمية تساعد الهيئة الطبية في إنجاز الدراسات والبحوث وتساعد الباحثين

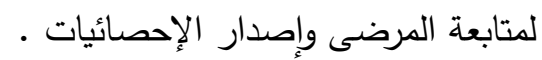

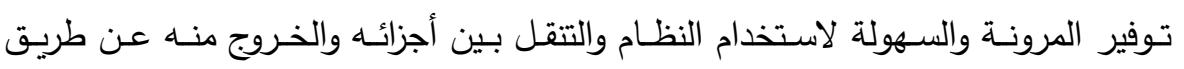

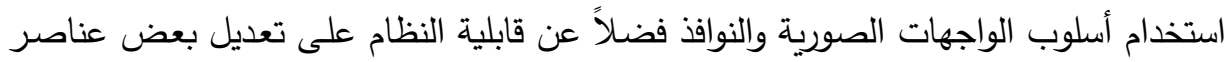
قاعدة المعلومات أو حذف عناصر أو إضافة عناصر جديدة دون التأثير في مجمل معلومات

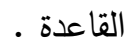
توفير الحماية للنظام للمحافظة على صحة المعلومات من أي تدخل غير مرغوب فيه. سرعة الاستجابة للاستفسارات المطلوبة. 


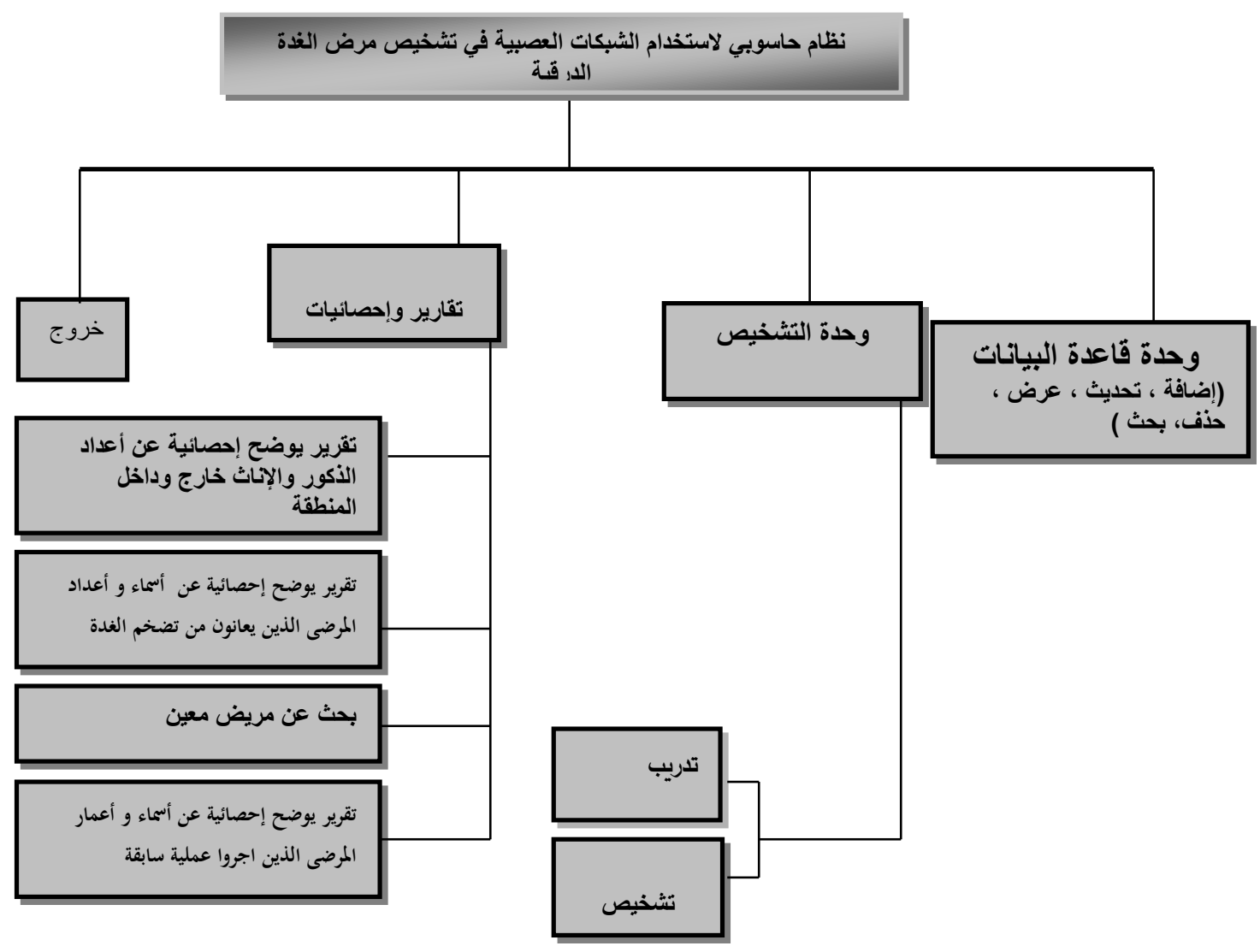

الشكل (3) المخطط العام لنظام الغدة الدرقية (thyroid) 


$$
\begin{aligned}
& \text { المصادر } \\
& \text { اسطيفان،جين جليل ،(1991). "التشخيص الطبي بمسـاعدة الحاسبة الإلكترونيـة"،مجلـة } \\
& \text { أبحاث الحاسوب، العدد السابع. } \\
& \text { الزبيدي ،لهيب محمد إبراهيم،(1998). "متابعة نظام طالب على مستوى الجامعة" ، مجلة } \\
& \text { أبحاث الحاسوب ،المجلد الثاني العدد الثاني. } \\
& \text { الصغير،د.علاء الدين،(1996 ) . "فرط نشاط الدرق سلسلة أمراض الغدد الصم" ، العدد } \\
& \text { الأول. } \\
& \text { علي،د.صباح عبد العزيز ،(1989) ـ "قواعد البيانات وأنظمة إدراتها" ،جامعة البصرة،دار }
\end{aligned}
$$

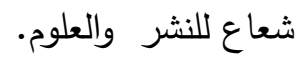

$$
\begin{aligned}
& \text { الناظر ،سائد محمود،(1997) "فيجول بيسك إصدار 5" كتاب المبرمج ،ط1،دار شعاع }
\end{aligned}
$$

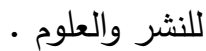

$$
\begin{aligned}
& \text { الوتار ، طرفة ياسين حامد ، (2000). "تمييز بصمات الأصابع باستخدام الثبكة العصبية }
\end{aligned}
$$

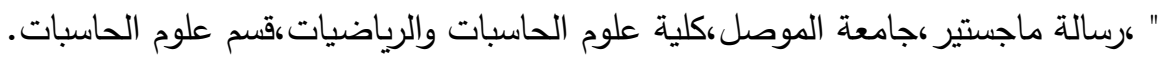

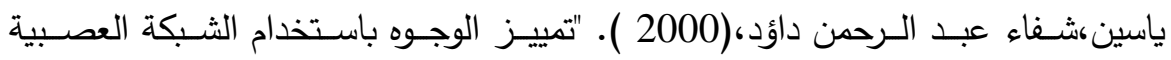

$$
\begin{aligned}
& \text { النيوكوكنترونية" ،رسالة ماجستير ،كلية الهندسة،قسم هندسة الحاسبات،جامعة الموصل. }
\end{aligned}
$$

[8] Dats.A., (1998) "The Use Of Computer -Assisted Diagnosis In Cardiac Perfusion Nuclear Medicine Studies" : 35:771-74.

[9] Demuth,H., Beate,M. (1998) "Neural ntework toolbox for use with Matlab", the math works Inc ,MA,USA.

[10] Eric, D ., patrick N. , (1995) "Neural networks", Macmillan.

[11] Kinnebrack, W., (1995) "Neural Networks Fundamentals Applications, Examples", Galgotia Publications.

[12] Lancet, A., (1995)"Application of Artificial Neural Network to clinical medicine", Pattern recognation, Vol. 346, P (1135).

[13] Roch,A.,F., Harbert,J.C.,(2000). ”Textbook of Nuclear Medicine clinical Application “.

[14] Werner, K., (1995) Neural Networks , Printic-hill.

[15] Zurada. J. M., (1996) "Introduction To Artificial Neural systems", Jacio Publishing House. 